\title{
Improved Ground-Based Remote-Sensing Systems Help Monitor Plant Response to Climate and Other Changes
}

\begin{abstract}
S cientists at the U.S. Geological Survey are improving and developing new ground-based remote-sensing instruments and techniques to study how Earth's vegetation responds to changing climates. Do seasonal grasslands and forests "green up" early (or late) and grow more (or less) during unusually warm years? How do changes in temperature and precipitation affect these patterns? Innovations in groundbased remote-sensing instrumentation can help us understand, assess, and mitigate the effects of climate change on vegetation and related land resources.
\end{abstract}

\section{Introduction}

Measuring plant phenology - that is, seasonal changes in plant growth-is an important part of climate research because it helps relate those seasonal vegetation changes to more regional changes in climate and environment. Remote-sensing imagery from satellites and aircraft is widely available, but it is limited because observations are infrequent and the image resolution is coarse. Oftentimes one pixel represents 900 square meters or more, so phenology observations are skewed where closely spaced plants have different seasonality - for example, in mountain meadows where seasonal grasses and evergreens live together. Scientists at the U.S. Geological Survey (USGS) are improving remote-sensing techniques by developing ground-based instruments

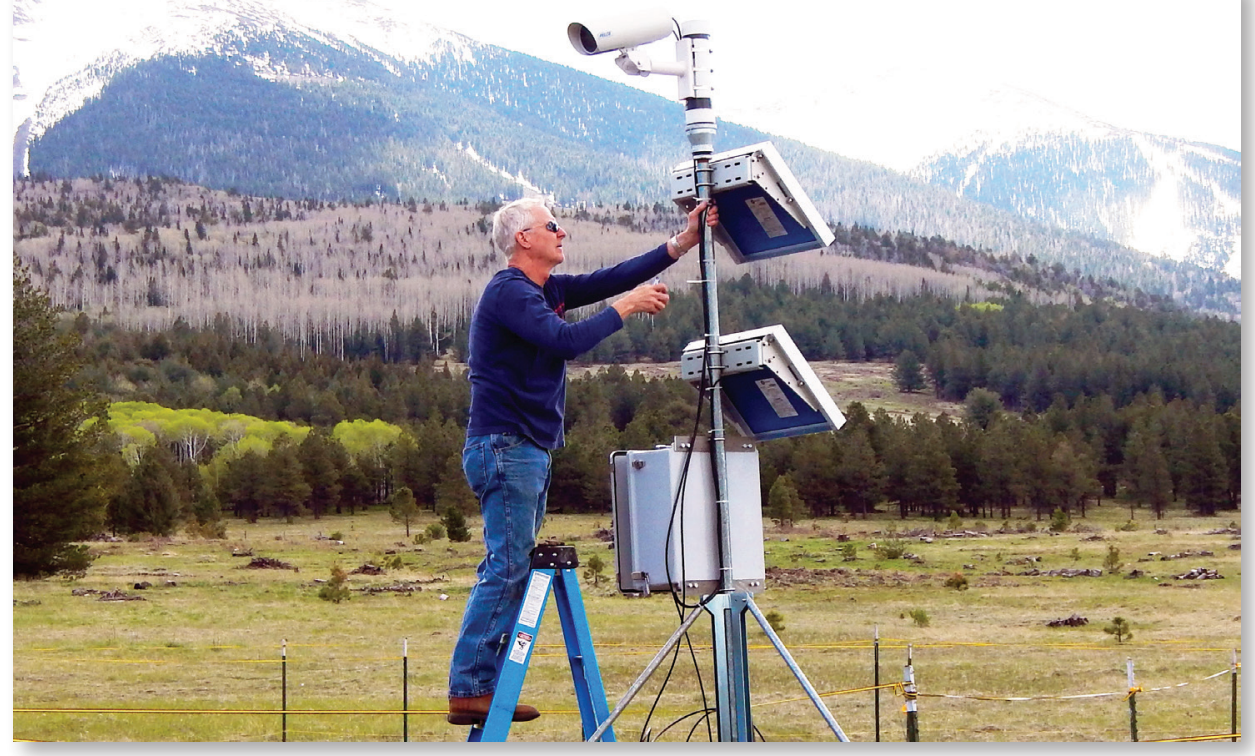

A High Dynamic Range Land Vegetation Imaging System (HDR-LVIS) is installed at the Hart Prairie Preserve near Flagstaff, Arizona, as part of a U.S. Geological Survey partnership with The Nature Conservancy. The HDR-LVIS is used to analyze how forest and meadow plants at this high-elevation site respond to a variable and changing climate. U.S. Geological Survey photograph by Dennis Dye.

that more accurately measure local phenology, as well as solar radiation. When combined with regional imagery, these new techniques reduce uncertainties in interpretation and help scientists and land managers study vegetation and climate across a broad range of ecosystems.

Improved USGS remote-sensing instruments use high dynamic range (HDR) imaging - a digital photography technique that is commonly used for practical and artistic purposes but is new in scientific remote-sensing applications. HDR imaging is used to record a range of brightness (both very bright and very dim) that is much wider than is possible with today's digital camera technology. Sensitivity to more light levels is achieved by combining multiple standard (low dynamic range) images captured in rapid succession with systematically increasing exposures, such that the combined set of images captures all brightness variations in the scene without saturation or underexposure. HDR techniques were applied to create two new remote-sensing systems, the

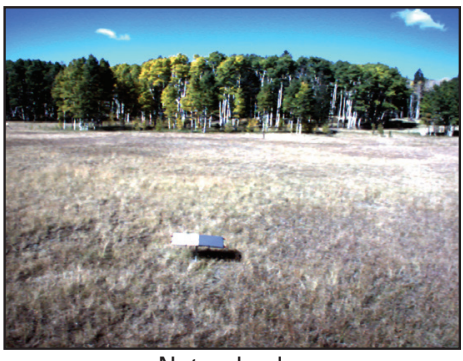

Natural color

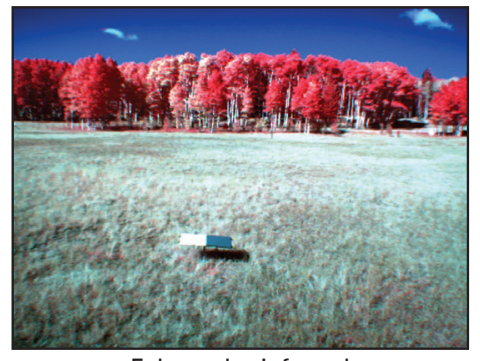

False color infrared
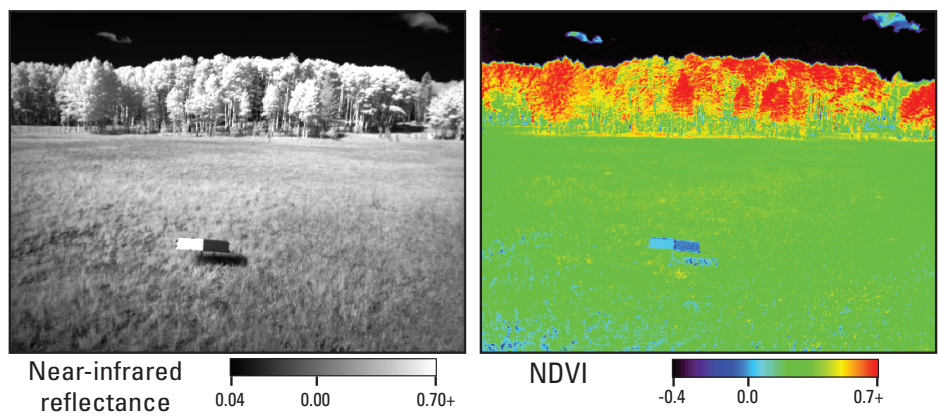

High dynamic range (HDR) multispectral images captured in October 2015 at the Nature Conservancy's Hart Prairie Preserve near Flagstaff, Arizona, by High Dynamic Range Land Vegetation Imaging System (HDR-LVIS) displayed as natural- and false-color infrared composites, near-infrared reflectance, and normalized-difference vegetation index (NDVI), which measures photosynthetic vigor and overall "greenness." U.S. Geological Survey images. 
High Dynamic Range Land Vegetation Imaging System (HDR-LVIS) and the High Dynamic Range All-Sky Imaging System (HDR-ASIS). Working in parallel, these remote-sensing systems provide an accurate snapshot of key factors that influence vegetation growth - the amount and directionality of sunlight from the sky and the capacity of vegetation in the landscape to intercept and absorb sunlight for photosynthesis.

\section{High Dynamic Range Land Vegetation Imaging System (HDR-LVIS)}

The HDR-LVIS instrument applies the HDR imaging technique to color (red, green, blue) and near-infrared cameras to measure solar energy reflected from plant leaves and provides accurate scientific-quality observations of landscape phenology. Unlike standard digital cameras, the instrument detects near-infrared wavelengths and accommodates large variations in brightness associated with clouds and shadowing. Images acquired simultaneously in visible and nonvisible (near-infrared) wavelengths are used to calculate for each pixel the fraction of incident radiation that is reflected from a plant leaf to the camera. These measurements are then used to calculate an index value that represents the density and vigor of vegetation in a landscape and its overall "greenness." Daily timeseries of HDR-LVIS images help USGS scientists detect, document, and understand local changes in vegetation phenology over seasons, years, and longer periods. For example, how active is vegetation after a rainfall event? The results, in turn, support our understanding of the broaderscale patterns of phenology and landscape changes observed from Earth-observing satellites.

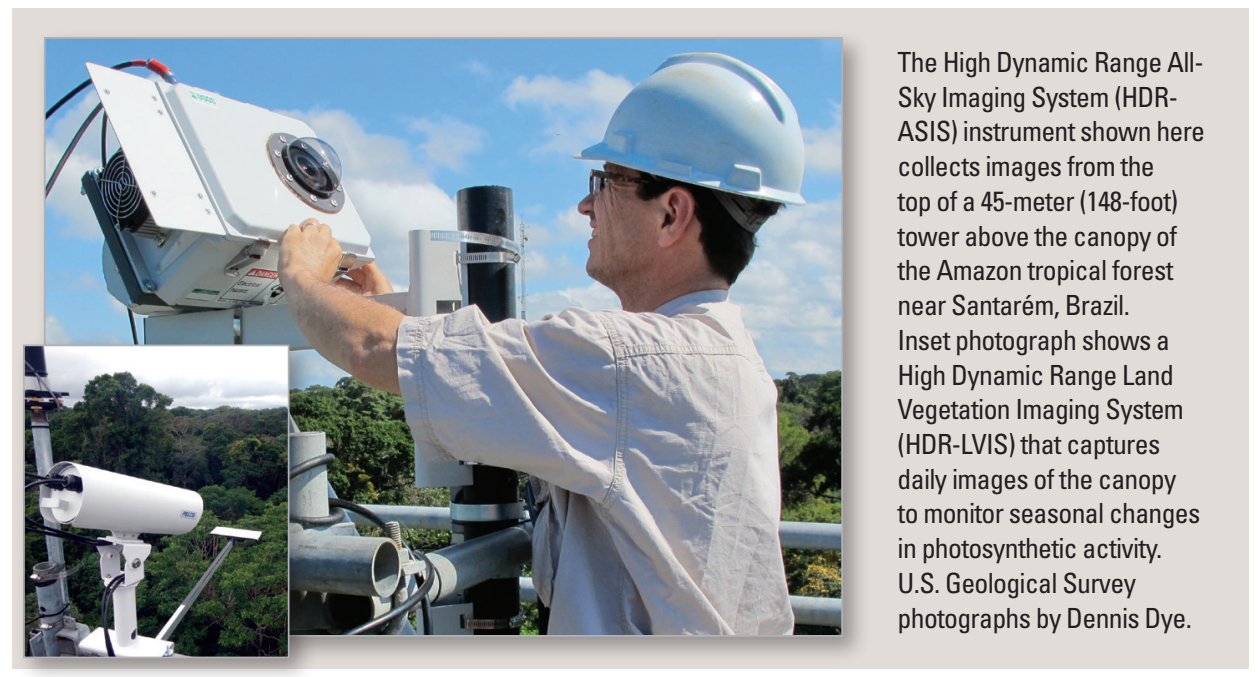

\section{High Dynamic Range All-Sky Imaging System (HDR-ASIS)}

The HDR-ASIS instrument

points upward at the sky and measures photosynthetically active radiation (PAR), that is, the radiation from the Sun that plants use for photosynthesis. Because HDR-ASIS uses HDR imaging techniques, it can record and monitor the amount of PAR received by the vegetation in a landscape from every angle in the sky, including intense PAR directly from the Sun. The resulting data are used to create computer-based simulations of vegetation photosynthesis to investigate how changes in atmospheric conditions, such as cloudiness or aerosols (like dust, smoke, or volcanic gases) affect solar radiation, and, in turn, vegetation growth. Since 2012, HDR-ASIS has been in near-continuous operation at the USGS science campus in Flagstaff, Arizona. Second-generation HDR-ASIS instruments were deployed in 2012 and 2015 at study sites in the Amazon region of Brazil as part of related studies of the seasonal growth dynamics of tropical forests, which are sponsored by the National Aeronautics and Space Administration (NASA) and the U.S. Department of Energy (DOE).

\section{Future Work}

\section{HDR-ASIS and HDR-LVIS}

instruments are currently operated near the USGS science campus in Flagstaff, Arizona, and at tropical forest sites in the Amazon region of Brazil, to collect photosynthetically active solar radiation and phenology data. Changes in climate and phenology will be analyzed as the time period of observations grows to several years and longer. Ground-based remotesensing techniques for plant phenology could be further improved by equipping existing instruments with real-time data reporting capabilities and by building a larger network of instruments to help improve understanding of climate change at regional and global scales. This ongoing work is part of USGS efforts to better understand the causes and consequences of land-use and climate change.

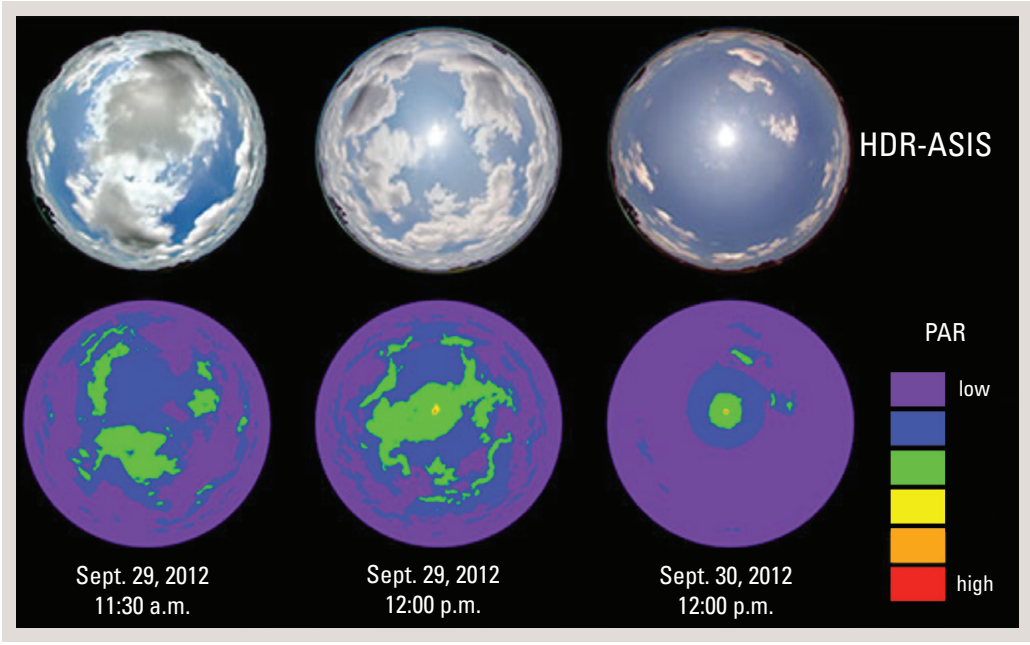

U.S. Geological Survey images showing observations from the High Dynamic Range All-Sky Imaging System (HDR-ASIS) at midday on two consecutive days at a study site in the Amazon tropical forest in Pará, Brazil. Sky condition images (top row) taken from upward-looking HDR-ASIS camera. Bright light in center of image is the Sun; white and gray colors show cloud cover. Colored plots (bottom row) correspond to images in top row and show how incoming light that is used by plants for photosynthesis is distributed across the sky. Photosynthetically active radiation, or PAR, is used to study how climate affects plant photosynthesis.
Dennis G. Dye and Rian C. Bogle Edited by Sarah E. Nagorsen Layout by Cory D. Hurd

For more information contact: Dennis G. Dye U.S. Geological Survey Western Geographic Science Center 2255 North Gemini Drive Flagstaff, AZ 86001-1637 ddye@usgs.gov http://geography.wr.usgs.gov

ISSN 2327-6916 (print) ISSN 2327-6932 (online) http://dx.doi.org/10.3133/fs20163013 